\title{
Transformation of Petri net Models by Matrix Operations
}

\author{
Juan-Ignacio Latorre-Biel ${ }^{1}$ Emilio Jiménez-Macías ${ }^{2}$ Juan Carlos Sáenz-Díez ${ }^{2}$ \\ Eduardo Martinez-Cámara ${ }^{2}$ \\ ${ }^{1}$ Department of Mechanical Energetic and Materials Engineering, Public University of Navarre, Spain, \\ juanignacio.latorre@unavarra.es \\ ${ }^{2}$ High Technical School of Industrial Engineering, University of La Rioja, Spain, \\ \{emilio.jimenez, julio.blanco, mercedes.perez\}@unirioja.es
}

\begin{abstract}
Petri nets constitute a modeling paradigm able to describe discrete event systems characterized by features such as parallelism, precedence, concurrence, and synchronization. Petri nets are applied extensively and successfully for modeling systems belonging to a broad range of fields. In this context, transformation of Petri net models constitutes a process with diverse applications, such as simplifying the model for developing structural analysis or for performance evaluation, as well as comparing different models, describing nets whose structure changes over time, or merging models with exclusive entities. The transformation of the structure of a Petri net can be carried out from different points of view. In this paper, this transformation is developed by means of matrix operations. A list of matrix operations is presented and the preservation of some significant properties of the Petri net is discussed as a practical tool for transforming Petri net models by operations in the incidence matrices.
\end{abstract}

Keywords: Petri nets, model transformation, matrixbased operations, alternative structural configuration, equivalence class

\section{Introduction}

Petri nets constitute a paradigm for modeling discrete event systems (DES). They are especially suited for DES characterized by features such as parallelism, precedence, concurrence, and synchronization. A Petri net model of a DES contains different elements for representing both the static structure and the dynamic behavior (Jiménez-Macías E. and Pérez-Parte, 2004; Latorre-Biel and Jimenez-Macias, 2011).

One of the well-known advantages of the Petri nets consists of a double complementary representation. On the one hand, the static structure of a Petri net can be described by means of a directed, weighted, and bipartite graph, whose nodes, called places and transitions, can be classified into two disjoint sets. In this graphical description the dynamics of the DES, and its state, is represented by tokens. On the other hand, the static structure of a Petri net can also be described by means of the input and output incidence matrices. Its state and the dynamic behavior may be represented by a marking or state vector, as well. This matrix-based description provides a quantitative representation of the DES able for structural analysis and performance evaluation (Murata, 1989).

By the application of matrix operations to the incidence matrix of a Petri net, it is possible to change the static structure of the net. This modification leads to a new model of the discrete event system, which may preserve certain properties of the original Petri net, depending on the matrix operation that has been applied. Many properties have been defined for Petri nets, such as equivalence, liveness, reachability, deadlockfreedom, reversibility, soundness, boundedness, controlled siphon property, consistency, conservativeness, persistence, coverability, repetitiveness, or fairness (Murata, 1989; Silva, 1993; Esparza and Nielsen, 1994). Informal descriptions of some of the mentioned properties are (Silva, 1993):

Boundedness. A Petri net is bounded if its set of reachable markings is finite.

Reachability. The reachability problem for Petri nets consists of deciding, given a Petri net $\left(N, M_{0}\right)$ and a marking $M$ of $N$, if $M$ can be reached from $M_{0}$.

Liveness. A Petri net is live if every transition can always occur again.

Deadlock-freedom. A Petri net is deadlock free if every reachable marking enables some transition (Silva, 1993). It is a weaker condition than liveness (Murata, 1989).

Home states. A marking of a Petri net is a home state if it is reachable from every reachable state. The home state problem consists in deciding given a Petri net $(N$, $M_{0}$ ) and a reachable marking $M_{0}$ if $M$ is a home state. The subproblem of deciding if the initial marking of a Petri net is a home state is the problem of deciding if a Petri net is cyclic (Silva, 1993) or reversible (Murata, 1989; Esparza and Nielsen, 1994).

Equivalence. Despite there are different kinds of equivalence, in this paper, the following approach will be followed: two Petri nets are said to be equivalent iff their reachability trees are isomorphous. This property is particularly interesting since it reflects that the Petri 
net with isomorphous reachability trees present similar behavior (Medina-Marin et al, 2013; Silva, 1981).

This form of defining equivalence implies the preservation of many properties, since one methodology to prove the verification of certain property by a Petri net consists of analyzing its reachability graph (Murata, 1989). As a consequence, it can be considered as a very useful property, since, for example, performance evaluation depends on the behavior of the system. Furthermore, decision-making support based on the simulation of a Petri net model, is closely related to performance evaluation and, eventually, to the behavior of the system (Silva, 1985).

Transformation of a Petri net constitutes a common process in some applications of this paradigm. For example, certain activities, such as performing structural analysis can be facilitated by the simplification of the Petri net model (Esparza and Nielsen, 1994).

In the case of complex Petri nets or simple Petri nets with complex behavior, calculating the reachable or coverability tree, as well as developing performance evaluation depends strongly on available power of computation. As a consequence, simplifying a model of a system may lead to the use of less computer resources, such as time and memory (Silva and Colom, 1988; Berthelot, 1987).

Matrix based operations may reduce the size of an incidence matrix or may allow comparing two different Petri nets, whose input and output incidence matrices are initially different. Knowing that two Petri nets are equivalent may simplify their analysis, since studying one of them might make unnecessary the analysis of the equivalent Petri net (Silva, 1981).

Moreover, techniques of top-down modeling can be implemented by transforming a low-detailed model into a more refined one (Esparza and Nielsen, 1994).

In order to develop the best transformation for a given purpose it is useful to be aware of the tools available for this task, as well as, which properties are preserved in the Petri net, after the transformation.

Efficient algorithms for transforming Petri net models are performed by modifying their incidence matrices. As a consequence, it constitutes a convenient tool for supporting these processes, taking into account a range of available matrix operations and the properties of the original Petri nets that they preserve (Silva, 1981; Latorre-Biel et al, 2015).

In this paper, some matrix operations applied to the incidence matrix of a Petri net, and the properties preserved in the process, between the original and the resulting Petri nets are discussed.

In the rest of the paper, the following contents are provided. Section 2 discusses the basic concepts of Petri nets. Section 3 discusses some operations and the properties they preserve. Next section addresses the conclusions and future research lines. Last section is related to the bibliographical references.

\section{Petri Nets}

Some basic concepts of Petri nets are given in this section with the purpose of introducing the reader in the subject and of providing with the notation that will be used in the following section. Moreover, some properties of Petri nets will be discussed. For more information on this topic see (Murata, 1989) and (Silva, 1993).

Following (Murata, 1989), a Petri net is a four-tuple $N=\square P, T$, Pre, Post $\square$, where, $P$ and $T$ are disjoint, finite, non-empty set of places and transitions respectively, Pre: $P \square T \rightarrow \mathbb{N}$ is the pre-incidence or input function, and Post: $T \square P \rightarrow \mathbb{N}$ is the prostincidence or output function.

The structure of a Petri net can be described by the pre-incidence and post-incidence matrices, $W^{-}$and $W^{+}$ respectively. In particular, their elements verify that $w_{i j}{ }^{+}$ $=\operatorname{Pre}\left(p_{i}, t_{j}\right)$ and $w_{k l}{ }^{-}=\operatorname{Post}\left(t_{l}, p_{k}\right)$. These values can also be called weight of the arc linking a place and a transition. It can be seen that $W^{-}, W^{+} \in M_{m} n$, where $m$ $=|P|$ and $n=|T|$. Every row of the pre-incidence and post-incidence matrices is associated to a different place of the Petri net, while every column is associated to a transition.

It is possible to define a single incidence matrix from the pre-incidence and post-incidence matrices:

$$
W=W^{+}-W^{-}
$$

There is a characteristic of the Petri net related to the usefulness of the incidence matrix $W$ for describing accurately the static structure of a Petri net. A Petri net is said to be pure if it does not contain any self-loop. Informally, a self-loop is a pair $\left\{p_{i}, t_{j}\right\}$, where $p_{i} \in P$ and $t_{j} \in T$, such that $p_{i}$ is input place and output place of the same transition $t_{j}$. In particular, it can be seen that if $\left\{p_{i}\right.$, $\left.t_{j}\right\}$ is a self-loop then $w_{i j}{ }^{+}=\operatorname{Pre}\left(p_{i}, t_{j}\right) \neq 0$ and $w_{i j}{ }^{-}=$ $\operatorname{Post}\left(t_{j}, p_{i}\right) \neq 0$.

Furthermore, given a Petri net $N=\square P, T$, Pre, Post $\square$, where $|P|=m$ and $|T|=n, \forall W \in M_{m} \quad n \exists ! W^{+}, W^{-} \in M_{m} n$ such that $W=W^{+}-W^{\text {iff }} W$ is pure. In other words, due to the fact that $W^{+}$and $W^{-}$represent the static structure of the Petri net, $W$ is an also valid representation of this structure iff $\nexists w_{i j} \in W$, such that $w_{i j}=w_{i j}{ }^{-}+w_{i j}{ }^{+}$, where $w_{i j}{ }^{+} \neq 0$ and $w_{i j} \neq 0$. Notice that otherwise $w_{i j}$ could be decomposed in infinite many positive integers associated to $w_{i j}{ }^{-}$and $w_{i j}{ }^{+}$.

As we have seen, self-loops reduce the usefulness of the incidence matrix for describing the static structure of a Petri net, since information is lost from $W^{+}, W^{-}$to $W$. In order to avoid this problem the self-loop can be removed by means of one of the following ways:

a) Introduce a new "dummy" place and transition in one of the arcs of the self-loop (Esparza and Nielsen, 1994). This solution is general, since it can be applied to any self-loop, but increases the size of the resulting incidence matrix. 
b) If one of the components of the pair that conforms the self-loop have not any additional input or output element, different from the other element of the pair, then, it can be removed. This means that the incidence matrix is reduced in one row or one column. This solution can be applied only to particular cases and it decreases the size of the resulting incidence matrix. Liveness, safeness, and boundedness are preserved (Esparza and Nielsen, 1994).

As a conclusion of the previous considerations, it can be stated that a non-pure Petri net can always be transformed in a Pure Petri net, likely modifying its dimensions. As a consequence, the structure of any Petri net can be described accurately by a single incidence matrix. This conclusion is important for the subsequent considerations on matrix operations applied to incidence matrices of Petri nets.

The previous considerations are related to the static structure of a Petri net. The consideration of its dynamic behavior can be addressed by means of the evolution of the state of the system, which is represented by means of the marking of the Petri net.

The marking of a Petri net can be defined as an application $M: P \rightarrow \mathbb{N}$, assigning a non-negative integer to every place of the net. The state of the Petri net can be represented by a marking or state vector, whose components are the marking associated to every place of the net, $M\left(p_{i}\right) \forall p_{i} \in P$.

As a consequence, a marked Petri net or Petri net system, can be defined as the pair $\square N, M_{0} \square$, where $N$ is a Petri net and $M_{0}$ is its initial marking.

\section{Petri Net Analysis}

A certain Petri net model can be studied for correctness, following a procedure of qualitative analysis. This approach may allow checking the verification of properties, such as the ones mentioned in the introduction, e.g. liveness, boundedness, or reachability.

There are several techniques for analyzing Petri net systems (Murata, 1989):

- Analysis by enumeration. Requires the construction of the reachability graph for bounded Petri nets or the coverability graph for unbounded Petri nets. With the mentioned graph, it is immediate to prove the verification of properties by the Petri net, such as liveness or reachability. However, the construction of this graph may be computationally costly, even unaffordable, due to the combinatorial state explosion problem.

- Analysis by transformation. This technique is based in the transformation of the Petri net model by the application of operations that preserve the properties expected to prove. This process is aimed at transforming the Petri net into another one, where it is easier to check the verification of certain properties. In fact, this objective may be achieved by arriving to a simpler model or to a Petri net, whose properties are already known.

- Structural analysis. In this case, several techniques of linear programming can be applied to deduce structural properties verified by the Petri net or ad hoc deductions can be performed based on graph-based techniques. As a result, some association between the structure of the Petri net and its dynamic behavior is determined.

- Analysis by simulation. This is the only technique that does not lead to exact results; hence, it cannot allow proving the verification of properties. However, by defining appropriate configurations for the freedom degrees of the Petri net, it is possible to acquire knowledge on the behavior of the system under certain conditions.

On the other hand, the evaluation of the efficiency of the Petri net model is important for certain applications, deducing from this analysis, the efficiency of the modeled discrete event system. This study can be carried out by means of techniques of quantitative analysis or performance evaluation, leading to the calculation of parameters that measure the quality of the system or its behavior, such as yield, costs, or utilization rate, just to give a few examples belonging to the field of manufacturing management.

A transformation of a Petri net model may require preserving certain properties. For example, if the purpose of the transformation is to simplify the model of the system for verifying its correctness, then preserving properties such as liveness or boundedness may be convenient. On the contrary, if the purpose of the transformation is to reduce the computational effort required to evaluate the efficiency of the Petri net, the transformation should lead to a simplified equivalent Petri net in terms of having an isomorphous reachability graph.

In the following section, several matrix operations that can be applied on the incidence matrix of a Petri net model will be presented, as well as some properties they preserve. As a result, a given transformation of a Petri net model could be performed by the application of some of the matrix operations to the incidence matrix of the net, regarding the properties that should be preserved. The implementation of matrix operations in an algorithm is a convenient form of transforming a Petri net in an efficient and automatic process.

\section{Matrix Operations}

Reference (Silva, 1981) discuss six matrix operations, three for rows and three for columns of the incidence matrix, applied to an incidence matrix; namely: 
- Adding a row (column) of zeros. The graphical interpretation consists of adding an isolated place (transition) to the Petri net. The dimension of the state or marking vector should be adjusted to the variation in the dimensions of the incidence matrix.

- Removing a row (column) of zeros. This operation can be interpreted as removing and isolated place or transition of the Petri net. Analogously to the previous operation,

- Swapping two rows (columns). The interpretation of this operation in the Petri net graph consists of interchanging the names of the places (transitions) associated to the swapped rows (columns) of the incidence matrix. The size of the incidence matrix keeps constant after the application of this operation.

These six matrix operations preserve the structure of the reachability graph (coverability tree for unbounded Petri nets), i.e. both the reachability (coverability) graph of the original and the transformed Petri nets are isomorphous.

As a consequence, it is immediate to prove that the properties of liveness, safeness, boundedness, reachability, reversibility, or equivalence are preserved by the application of one of these matrix operations, as well as of any of their feasible combinations.

A significant effort has already been devoted to the development of reduction rules for Petri nets (Murata, 1989; Esparza and Nielsen, 1994). The application of six of them are discussed from the point of view of matrix operations (Latorre-Biel et al, 2015). These six reduction rules lead to some additional elementary matrix operations that can be applied to an incidence matrix for transforming the Petri net model. Any of these six reduction rules preserve properties such as liveness, safeness, and boundedness (Esparza and Nielsen, 1994). As a consequence, the associated matrix operations also preserve these properties. In particular, it can be considered the following matrix operations:

- Sum of two rows $q$ and $r$ that verify the properties mentioned below. The resulting row is included in the incidence matrix, while the original rows $q$ and $r$ are removed, as well as the column $i$, which is also removed. Notice that $i$ is defined in the first of the following properties:

○ i) $\exists$ ! $i \in \mathbb{N}$, where $1 \leq i \leq n=|T|$, such that $w_{q i} \supsetneqq 0 \wedge w_{r i} \varsubsetneqq 0$.

○ ii) $\forall j \in \mathbb{N}$, where $1 \leq j \leq n=|T|$ and $j \neq$ $i$, it is verified that $w_{q j}=0 \vee w_{r j}=0$.

○ iii) $\forall k \in \mathbb{N}$, where $1 \leq k \leq m=|P|$ and $k \neq q \wedge k \neq r$, then $w_{k i}=0$.

This operation is based in the reduction rule of fusion of series places or FSP (Esparza and Nielsen, 1994), which is a particular case of the macroplace rule (Latorre-Biel et al, 2014 ; Murata, 1989). Property (i) justifies the existence of a single common transition between the series places. Property (ii) guarantees that the matrix operation does not add any self-loop to the Petri net. In other words, if the original Petri net is pure the matrix operation will lead to a resulting Petri net, which is also pure. Property (iii) addresses the fact that the intermediate transition $t_{i}$ is only linked to the series places. Notice that the column $i$ is associated to the transition $t_{i}$.

Analogously it can be stated the following matrix operation:

- Sum of two columns $q$ and $r$ that verify the properties mentioned below. The resulting column is included in the incidence matrix, while the original columns $q$ and $r$ are removed, as well as the row $i$, which is also removed. Notice that $i$ is defined in the first of the following properties:

○ i) $\exists ! i \in \mathbb{N}$, where $1 \leq i \leq m=|P|$, such that $w_{i q} \supsetneqq 0 \wedge w_{i r} \varsubsetneqq 0$.

○ $i i) \forall j \in \mathbb{N}$, where $1 \leq j \leq m=|P|$ and $j$ $\neq i$, it is verified that $w_{j q}=0 \vee w_{j r}=0$.

○ iii) $\forall k \in \mathbb{N}$, where $1 \leq k \leq n=|T|$ and $k$ $\neq q \wedge k \neq r$, then $w_{i k}=0$.

The previous matrix operation is based in the reduction rule of fusion of series transitions or FST (Esparza and Nielsen, 1994), which is a particular case of the transition fusion rules (Latorre-Biel and JiménezMacías, 2013; Murata, 1989). Property (i) justifies the existence of a single common place between the series transitions. Property (ii) guarantees that the matrix operation does not add any self-loop to the Petri net. Property (iii) addresses the fact that the intermediate place $p_{i}$ is only linked to the series transitions. Notice that the row $i$ is associated to the place $p_{i}$.

- Removing a row $q$ if the properties mentioned below are verified:

○ i) $\exists j, k \in \mathbb{N}$, where $1 \leq j, k \leq n=|T|$, such that $w_{q j} \geqq 0 \wedge w_{q k} \ggg 0$.

○ ii) $\exists ! r \in \mathbb{N}$, where $1 \leq r \leq m=|P|$ and $\exists j, k \in \mathbb{N}$, where $1 \leq j, k \leq n=|T|$, such that $w_{r j} \supsetneqq 0 \wedge w_{r k} \supseteqq 0$.

○ iii) $\forall i \in \mathbb{N}$, where $1 \leq i \leq n=|T|$ and $i$ $\neq j \wedge i \neq k$, then $w_{q i}=0 \wedge w_{r i}=0$.

This operation is based in the reduction rule of fusion of parallel places or FPP (Esparza and Nielsen, 1994), which is a particular case of the implicit place rule (Latorre-Biel and Jiménez-Macías, 2013b; Latorre-Biel and Jimenez-Macias, 2011). Property (i) justifies the existence of an input and an output transition for the place $p_{q}$, associated to the qth row. Analogously, property (ii) guarantees the existence of an input and an output transition for the place $p_{r}$, associated to the $r t h$ row, which are the same as in case of place $p_{q}$. Property (iii) addresses the fact that there is a single input and 
output transitions for both, places $p_{q}$ and $p_{r}$. See figure 1 for a graphical representation.

Analogously it can be stated the following matrix operation:

- Removing a column $q$ if the properties mentioned below are verified:

○ i) $\exists j, k \in \mathbb{N}$, where $1 \leq j, k \leq m=|P|$, such that $w_{j q} \supsetneqq 0 \wedge w_{k q} \lessgtr 0$.

○ ii) $\exists ! r \in \mathbb{N}$, where $1 \leq r \leq n=|T|$ and $\exists$ $j, k \in \mathbb{N}$, where $1 \leq j, k \leq m=|P|$, such that $w_{j r} \supsetneqq 0 \wedge w_{k r} \supsetneqq 0$.

○ iii) $\forall i \in \mathbb{N}$, where $1 \leq i \leq m=|P|$ and $i$ $\neq j \wedge i \neq k$, then $w_{i q}=0 \wedge w_{i r}=0$.

This operation is based in the reduction rule of fusion of parallel transitions or FPT (Esparza and Nielsen, 1994), which is a particular case of the identical transition rule (Latorre-Biel and Jiménez-Macías, 2013; 2013b). Property (i) justifies the existence of an input and an output place for the transition $t_{q}$, associated to the qth column. Analogously, property (ii) guarantees the existence of an input and an output place for the transition $t_{r}$, associated to the $r$ th column, which are the same as in case of transition $t_{q}$. Property (iii) addresses the fact that there is a single input and output places for both, transitions $t_{q}$ and $t_{r}$.

It should be mentioned that the opposite matrix operation to the two previously mentioned ones might also been applied: the addition of a row or a column, when there is another one composed of zeros but two elements which present the same absolute value but opposed signs. The added row or column should present the same elements and in the same positions than the one that should be already present in the incidence matrix before the operation. As well as in the previous operations, the properties of liveness, safeness, and boundedness are also preserved.

In particular, it is possible to present the following two matrix operations:

- Adding a row $q$ if the properties mentioned below are verified:

○ i) $\exists j, k \in \mathbb{N}$, where $1 \leq j, k \leq n=|T|$, such that $w_{q j} \gtreqless 0 \wedge w_{q k} \lesseqgtr 0$.

○ ii) $\exists ! r \in \mathbb{N}$, where $1 \leq r \leq m=|P|$ and $\exists j, k \in \mathbb{N}$, where $1 \leq j, k \leq n=|T|$, such that $w_{r j} \gtreqless 0 \wedge w_{r k} \lesseqgtr 0$.

○ iii) $\forall i \in \mathbb{N}$, where $1 \leq i \leq n=|T|$ and $i$ $\neq j \wedge i \neq k$, then $w_{q i}=0 \wedge w_{r i}=0$.

This operation is based in the opposite process to the reduction rule of fusion of parallel places or FPP (Esparza and Nielsen, 1994).

- Adding a column $q$ if the properties mentioned below are verified:

○ i) $\exists j, k \in \mathbb{N}$, where $1 \leq j, k \leq m=|P|$, such that $w_{j q} \supsetneqq 0 \wedge w_{k q} \supseteqq 0$.
○ ii) $\exists ! r \in \mathbb{N}$, where $1 \leq r \leq n=|T|$ and $\exists$ $j, k \in \mathbb{N}$, where $1 \leq j, k \leq m=|P|$, such that $w_{j r} \gtreqless 0 \wedge w_{k r} \supseteqq 0$.

○ iii) $\forall i \in \mathbb{N}$, where $1 \leq i \leq m=|P|$ and $i$ $\neq j \wedge i \neq k$, then $w_{i q}=0 \wedge w_{i r}=0$.

This operation is based in the opposite process to the reduction rule of fusion of parallel transitions or FPT (Esparza and Nielsen, 1994).

The multiplication and the division of elements of an incidence matrix can be useful for transforming a Petri net. Moreover, this operation, as well as the other ones presented in this paper can be applied in sequence to profit from the combination of their effects.

One of the matrix operations that will be analyzed in this section is presented below:

- Multiplying a row $q$ by a positive integer $k$. This operation should comply with an additional restriction described in the following. Let us call $M_{0}\left(p_{k}\right)$ the initial marking of the place $p_{k}$, associated to the $q$ th column in the original Petri net. Let us call $M_{0}{ }^{\prime}\left(p_{k}\right)$ the initial marking of $p_{k}$ in the Petri net that results from the transformation. It should be verified that:

$$
M_{0}{ }^{\prime}\left(p_{k}\right)=k \cdot M_{0}\left(p_{k}\right)
$$

As it has been shown, the application of this matrix operation requires the multiplication of both, a row of the incidence matrix and the initial marking of the place associated to the multiplied row. Graphical interpretation.

It can be proven that the application of this matrix operation preserves the properties of liveness, safeness, and boundedness. This conclusion is immediate when it is realized that the reachability graph (coverability graph if the Petri net is unbounded) is isomorphous in the original and the transformed Petri net.

One of the matrix operations that will be analyzed in this section is presented below:

- Dividing a row $q$ by a positive integer $k$. This operation should comply with two additional restrictions described below. Let us call $M_{0}\left(p_{k}\right)$ the initial marking of the place $p_{k}$, associated to the qth column in the original Petri net. Let us call $M_{0}{ }^{\prime}\left(p_{k}\right)$ the initial marking of $p_{k}$ in the Petri net that results from the transformation. It should be verified that:

$$
\begin{aligned}
& \text { i) } M_{0}{ }^{\prime}\left(p_{k}\right)=M_{0}\left(p_{k}\right) / k \in \mathbb{Z} \\
& \text { ○ } \text { ii) } \forall i \in \mathbb{N} \text {, where } 1 \leq i \leq n=|T| \text { it is } \\
& \text { verified that } w_{q i} \in \mathbb{Z}
\end{aligned}
$$

As it has been shown, the application of this matrix operation requires the division of both, a row of the incidence matrix and the initial marking of the place associated to the multiplied row. The results of these two divisions should be integers, no matter if positive, negative, or zeros. 
It can be proven that the application of this matrix operation preserves the properties of liveness, safeness, and boundedness. This conclusion is immediate when it is realized that the reachability graph (coverability graph if the Petri net is unbounded) is isomorphous in the original and the transformed Petri net.

As an example of combined application of matrix operations, to appreciate the advantages of this approach, it can be considered the following matrix operations "dividing a row $q$ by a non-negative integer $k$ " and "removing a column $q$ " it can be considered the following one.

Let us consider the case presented in figure 1. In principle, the reduction rule of fusion of parallel places or FPP is not applicable, since the weight of the arcs of $p_{q}$ is $a$ and are different from the weight of the arcs of $p_{r}$ , which is $b$.

As a consequence, it is possible to divide the $q$ th row of the incidence matrix by $b$. As it can be seen in figure 1 , this row presents only two elements different to zero, whose value is $w_{q j}=b=w_{q k}$. Dividing this row by $b$ will lead to a new qth row, where the zeros are the same, while the other two elements different to zero, in the resulting row present a value of 1 .

As a second step, it is possible to apply again the rule of dividing the row $r$ by $a$, leading to a $r$ th row with zeros, with the exception of $w_{r j}=1=w_{r k}$.

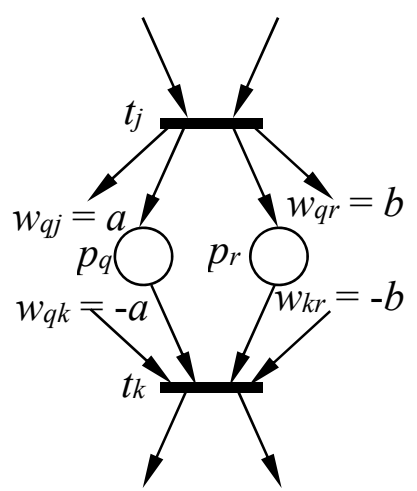

Figure 1. Graphical representation of the initial Petri net in an example of combined application of two matrix operations.

The situation of the Petri net after the application of these two matrix operations can be seen in figure 2 .

Now it is possible to apply the matrix operation "removing a row $q$ ", since the conditions for the application of this matrix operation are complied.

As a result of this example of application, the size of the Petri net has been decreased in one row, and another row has reduced the values of its elements from $b \in \mathbb{Z}$ to 1.

\section{Conclusions}

The research line presented in this document has analyzed so far a set of 14 matrix operations for their potential application to the incidence matrix of a Petri net. The application of a sequence of these operations to the incidence matrix of a Petri net allow obtaining another Petri net that preserve certain properties of the initial one.

Special emphasis has been place on the preconditions and the consequences of their application, as well as the properties of the Petri net they preserve. Some of these matrix operations are based on previously developed reduction rules for Petri nets.

These operations can be applied easily in algorithms focused on the transformation of Petri nets for different purposes, such as net analysis, reduction of computer effort devoted to simulating the behavior of the Petri net, the comparison of the incidence matrices of different Petri nets to estimate their similarity, or to merge them into a single compound Petri net, just to give some examples.

As future research lines, it can be considered to increase the number of matrix operations to broaden the range of tools for Petri net transformations, as well as to analyze the preservation of other properties in the already presented matrix operations.

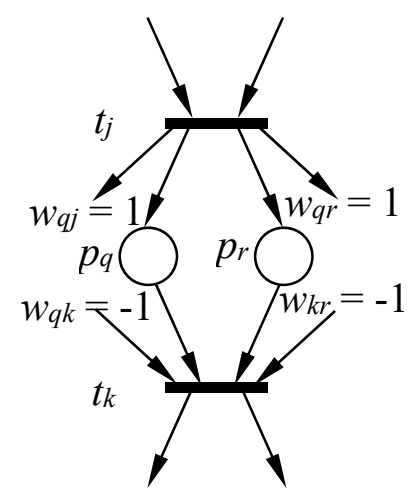

Figure 2. Graphical representation of the final Petri net in an example of combined application of two matrix operations.

\section{References}

G. Berthelot. Transformations and decompositions of nets. In Petri Nets: Central Models and their Properties. LNCS 254. W. Brauer, W. Reisig, and G. Rozenberg, Eds. Springer-Verlag, pp. 359-376, 1987.

J. Esparza, and M. Nielsen. Decidability Issues for Petri Nets. Technical Report, BRICS RS948, BRICS Report Series, Department of Computer Science, University of Aarhus, 1994. 
E. Jiménez-Macías, and M. Pérez-Parte. Simulation and optimization of logistic and production systems using discrete and continuous Petri nets. Simulation, 80(3):143152, 2004.

J. I. Latorre-Biel, E. Jiménez Macías, J. L. García-Alcaraz, J. C. Sáenz-Díez Muro, M. Pérez de la Parte. Alternatives aggregation petri nets applied to modular models of discrete event systems". In Proceedings of the European Modelling and Simulation Symposium. EMSS 2015, pages 465-470, Bergeggi, Italy, 2015.

J.I. Latorre-Biel, and E. Jimenez-Macias. Matrix-Based Operations and Equivalent Classes in Alternative Petri nets. In Proceedings of the European Modelling and Simulation Symposium EMSS 2011, pages 587-592, Rome, 2011.

J.I. Latorre-Biel, and E. Jiménez-Macías. Efficient Methodology For High Level Decision Making On A Manufacturing Facility". In Proceedings of the 8th EUROSIM Congress on Modelling and Simulation, pages 345 - 350, Cardiff (United Kingdom), 2013.

J.I. Latorre-Biel, and E. Jiménez-Macías. Simulation-based optimization of discrete event systems with alternative structural configurations using distributed computation and the Petri net paradigm. Simulation, 89(11):1310-1334, 2013.
J.I. Latorre-Biel, E. Jiménez-Macías, J. Blanco-Fernández, E. Martínez-Cámara, J.C. Sánez-Díez, and M. Pérez-Parte. Design and operation of a dairy plant by means of a decision support tool based on the Petri nets paradigm. In Proceedings of the European Modelling and Simulation Symposium EMSS 2014, pages 588-593, Bordeaux, 2014

J. Medina-Marin, J. C. Seck-Tuoh-Mora, N. HernandezRomero, and J. C. Quezada-Quezada. Petri net reduction rules through incidence matrix operations. In Proceedings of the European Modelling and Simulation Symposium EMSS 2013, pages 496-503, Athens, 2013.

T. Murata. Petri nets properties analysis and applications. In Proceedings of the IEEE 77(4):541-580, 1989. doi: $10.1109 / 5.24143$

M. Silva, and J. M. Colom. On the computation of structural synchronic invariants in $\mathrm{P} / \mathrm{T}$ nets. In Advances in Petri nets'88 LNCS 340, G. Rozenberg, Ed. Springer-Verlag, pp. 386-417, 1988.

M. Silva. Introducing Petri nets, In Practice of Petri Nets in Manufacturing, F. Di Cesare, (editor), pages 1-62. Ed. Chapman\&Hall, 1993.

M. Silva. "Las redes de Petri en la Automática y la Informática", translated as Petri nets in automatics and computation. Ed. AC, Madrid, 1985.

M. Silva. Sur le concept de macroplace et son utilisation pour l'analyse des reseaux de Petri'. RAIRO-Systems Analysis and Control, 15(4):57-67, 1981. 